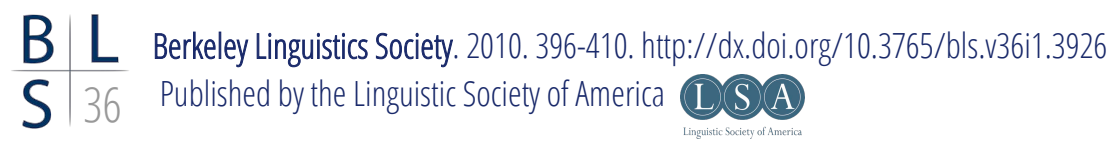

\title{
A Cross-linguistic Study of Sound Symbolism: The Images of Size
}

\author{
KAZUKO SHINOHARA ${ }^{1}$, SHIGETO KAWAHARA ${ }^{2}$ \\ ${ }^{1}$ Tokyo University of Agriculture and Technology; ${ }^{2}$ Rutgers University
}

\section{Introduction}

Although the sound-meaning relationship is often arbitrary (Saussure 1916), cases exist in which some sounds correspond to certain meanings. Such association between sounds and meanings is known as sound symbolism, and there has been a longstanding interest in the existence and the nature of sound symbolism. ${ }^{1}$ This paper reports an experiment on size-related sound symbolism, which shows that certain sound symbolisms hold robustly across languages. In particular, we investigate how the images of size (small or large) are affected by three phonetic factors: the height of vowels, the backness of vowels, and voicing in obstruents. Our rating experiment of four languages - Chinese, English, Japanese, and Korean - shows that these three factors contribute to the images of size, with only a few exceptions. To explain the results, we offer phonetic grounding of these size-related sound symbolic patterns. We further raise the possibility that these phonetically grounded sound symbolic patterns are 'embodied' in the sense of Johnson (1987) and Lakoff and Johnson (1980, 1999).

\section{$1 \quad$ Background}

Sound symbolism refers to cases in which particular images are associated with certain sounds; for example, Sapir's (1929) seminal experimental work shows that English speakers tend to associate [a] with an image larger than that associated with [i]. Previous studies have argued that these sound symbolic patterns have phonetic bases (e.g. Eberhardt 1940; MacNeilage and Davis 2001; Ohala 1983b, 1994; Paget 1930; Sapir 1929); for example, [a] may be perceived as larger than [i] because [a] involves wider opening of the mouth than [i] (see section 5 for

\footnotetext{
${ }^{1}$ There is a large body of literature on sound symbolism, which is too large to list in this short paper. For a recent summary of bibliographies on sound symbolism, see Akita (2009).
} 
more discussion on the phonetic grounding of sound symbolism). Building on this tradition of research, our paper addresses the following three questions: (i) whether sound symbolic patterns hold robustly cross-linguistically, and if so (ii) how, and (iii) why.

Before we proceed, we clarify why studying sound symbolism is important for (cognitive) linguistic theories. First, sound symbolism possibly constitutes a counterargument against the thesis of arbitrariness, i.e. arbitrary relations between a signifiant and a signifié (Saussure 1916). Second, to the extent that sound symbolic connections between sound and meaning may have phonetic bases, sound symbolism may instantiate a case of iconicity (Haiman 1983, 1985a, b) between sound and meaning: phonetic factors affect—or shape - meanings. Third, again to the extent that sound symbolism has phonetic bases, it may also constitute an instance of embodiment (Johnson 1987, Lakoff and Johnson 1980, 1999), which is one of the central tenets of cognitive linguistics. In short, sound symbolic patterns are, as we will argue, semantic patterns grounded in phonetic gestures (or their acoustic consequences); in other words, sound symbolic patterns are cases in which speakers reflect their phonetic behaviors upon the meaning of certain sounds. For these reasons we take the study of sound symbolism to be an interesting topic of linguistic theories.

\section{Research Questions}

Many researchers have pointed out some existing relations between sounds and the image of size. For example, Sapir (1929) showed that given two nonce words [mil] and [mal] and two tables (small and large), English speakers tend to associate [mal], not [mil], with a large table: [a] evokes a larger image than [i] for English speakers. Building on this observation, this paper addresses three questions. The first issue is whether this size-related sound symbolism holds across languages, beyond English. Building on previous work, our current experiment shows that it does (see also Ultan 1978 for a cross-linguistic lexical study).

Second, this paper explores exactly which phonetic dimensions determine the image of size. Previous researchers have offered different answers to this question. Some previous studies suggested that it is vowel height that determines the images of size (Kawahara et al. 2005). Others found that back vowels are perceived as larger than front vowels: for example, Newman (1933) found that English speakers judge all back vowels to be larger than all front vowels. Ultan (1978) argued that both height and backness affect the images of size. Furthermore, in addition to vocalic differences, Newman (1933) found that English speakers consider voiced obstruents to be larger than voiceless obstruents. In short, it remains controversial as to which phonetic factors determine the images of size. The second aim of this project is to address this question. Our experiment shows that all of the three factors - vowel height, backness, and obstruent voic- 


\section{A Cross-linguistic Study of Sound Symbolism}

ing - affect the image of size, albeit to different degrees in different languages.

The final issue concerns phonetic grounding of sound symbolism. Some scholars have suggested that size-related sound symbolism is grounded on the size of the oral cavity (e.g. Berlin 2006, Paget 1930, Sapir 1929). Building on these proposals, we attempt to clarify phonetic grounding of the size-related sound symbolic patterns. We further raise the possibility that the phonetically grounded sound symbolism instantiates a case of embodiment (Johnson 1987, Lakoff and Johnson 1980, 1999).

\section{$3 \quad$ Method}

The current study is a questionnaire-based rating experiment, in which the participants rated the size of various nonce words. To test whether sound symbolism holds cross-linguistically, we tested speakers of Chinese, English, Japanese, and Korean. The stimuli consisted of 40 disyllabic nonce words with VCVC form, in which the two vowels and the two consonants were identical (e.g. ibib). The consonants were four voiced obstruents $[\mathrm{b}, \mathrm{d}, \mathrm{g}, \mathrm{z}]$ and four corresponding voiceless obstruents $[\mathrm{p}, \mathrm{t}, \mathrm{k}, \mathrm{s}]$. The vowels were $[\mathrm{i}, \mathrm{u}, \mathrm{e}, \mathrm{o}, \mathrm{a}]$, which are the five vowels that all the target languages have. These factors were fully crossed (2 voicing types $* 4$ types of obstruents $* 5$ vowels) as in Table 1 . All of these words are nonce words in all the target languages.

(1) Table 1. List of stimuli.

\begin{tabular}{|l|l|l|l|l|}
\hline Voiced & \multicolumn{1}{|c|}{ b } & \multicolumn{1}{c|}{ d } & \multicolumn{1}{c|}{ g } & \multicolumn{1}{c|}{ z } \\
\hline \hline i & ibib & idid & igig & iziz \\
\hline u & ubub & udud & ugug & uzuz \\
\hline e & ebeb & eded & egeg & ezez \\
\hline o & obob & odod & ogog & ozoz \\
\hline a & abab & adad & agag & azaz \\
\hline & & & & \\
\hline Voiceless & \multicolumn{1}{|c|}{ p } & t & k & s \\
\hline \hline i & ipip & itit & ikik & isis \\
\hline u & upup & utut & ukuk & usus \\
\hline e & epep & etet & ekek & eses \\
\hline o & opop & otot & okok & osos \\
\hline a & apap & atat & akak & asas \\
\hline
\end{tabular}

The participants were 20 Chinese (Mandarin) speakers, 22 English speakers, 42 Japanese speakers, and 19 Korean speakers. In the experiment, participants were 
presented with the stimuli on a written questionnaire ${ }^{2}$ and asked to rate the size of each nonce word on a 1-4 scale ( $1=$ very small, $2=$ relatively small, $3=$ relatively large, $4=$ very large). They were instructed to imagine an exotic language in which the stimuli were spoken, and speculate on the meanings of these words, as in the instructions in (2). A sample question is shown in (3).

(2) Instructions:

Imagine an exotic language that you don't know. The language has a rich lexical inventory of adjectives that express a variety of "largeness" or "smallness". Now, a speaker of this language looks inside a box and finds a jewel. She verbally expresses how large or small it looks using one of these adjectives. Your task is to read each of the following words and guess its meaning - i.e., how large or small it is.

(3) Sample question:

ibib

1

2

very small relatively small

3

relatively large
4

very large

The stimuli were presented using Roman alphabet in a randomized order.

To assess the results statistically, a mixed linear model (Baayen 2008, Chapter 7) was used in which the main fixed factors were (i) vowel height, (ii) vowel backness, and (iii) voicing of obstruents. We also included additional fixed factors (place and continuancy) to distinguish different consonants and to soak up variability. For expository reasons, these two factors are not discussed in this paper. The model also included the speaker as a random factor. The degrees of freedom were assumed to be $n-2$ following the suggestion of Baayen (2008, p.248). After the general analysis, we carried out post-hoc analyses comparing three levels of height. To avoid the inflation of type I error, no multiple comparisons between each of the five vowels were conducted.

\section{$4 \quad$ Results}

Figure 1 shows the average ratings of all the five vowels in all four languages. Here and throughout, the error bars represent $95 \%$ confidence intervals. Although there is cross-linguistic variation, we observe some consistent patterns; for example, [i] is consistently rated as smaller than other vowels; [a] and [o] are generally rated as larger than other vowels.

\footnotetext{
${ }^{2}$ For Japanese participants, the first author pronounced these stimuli. We were particularly concerned about our Japanese participants assigning "Japanized reading" of alphabets on our stimuli. For a possible impact of reading on sound symbolism, see Kunihira (1971).
} 


\section{A Cross-linguistic Study of Sound Symbolism}

(4) Figure 1. Overall results. The error bars represent 95\% confidence intervals.

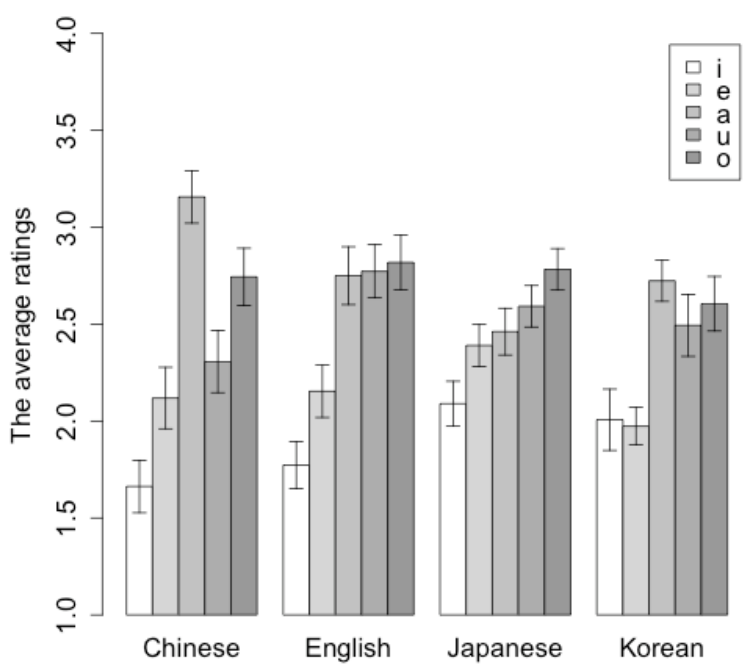

Figure 2 shows the effects of height on the image of size. Within each language, the left bar shows high vowels, the middle bar shows mid vowels, the right bar shows low vowels. There is a general trend in which the lower the vowel, the larger the image. The effects of height in the general linear mixed model are as follows: Chinese, $t(798)=9.48, p<.001$; English, $t(878)=1.74, p<.05$; Japanese, $t(1678)=.25$, n.s.; Korean, $t(758)=1.95, p<.05$.

Post-hoc comparisons of each level of height reveal that, first of all, mid vowels evoked significantly larger images than high vowels in Chinese and Japanese but not in the other two languages (Chinese, $t(638)=4.19, p<.001$; English, $t(702)=-.86$, n.s.; Japanese, $t(1342)=-5.00, p<.001$; Korean, $t(606)=1.25$, n.s.). Second, low vowel evoked larger images than mid vowels in Chinese and English to a statistically significant degree (Chinese $t(478)=6.32, p<.001$; English, $t(526)=5.34, p<.001)$. Japanese showed a significant reversal $(t(1006)=5.04$, $p<.001)$; Korean showed no significant differences $(t(454)=.6, n$.s. $)$.

Therefore, only one significant reversal to the height-size correlation is found in the behavior of Japanese speakers, who rated mid vowels to be larger than low vowels. This result contradicts our previous study (Kawahara et al. 2005), which found that Japanese speakers rate mid vowels to be smaller than low vowels. We do not have a good explanation of why we find this reversal in the current experiment. 


\section{Kazuko Shinohara and Shigeto Kawahara}

(5) Figure 2. The effect of vowel height on size ratings.

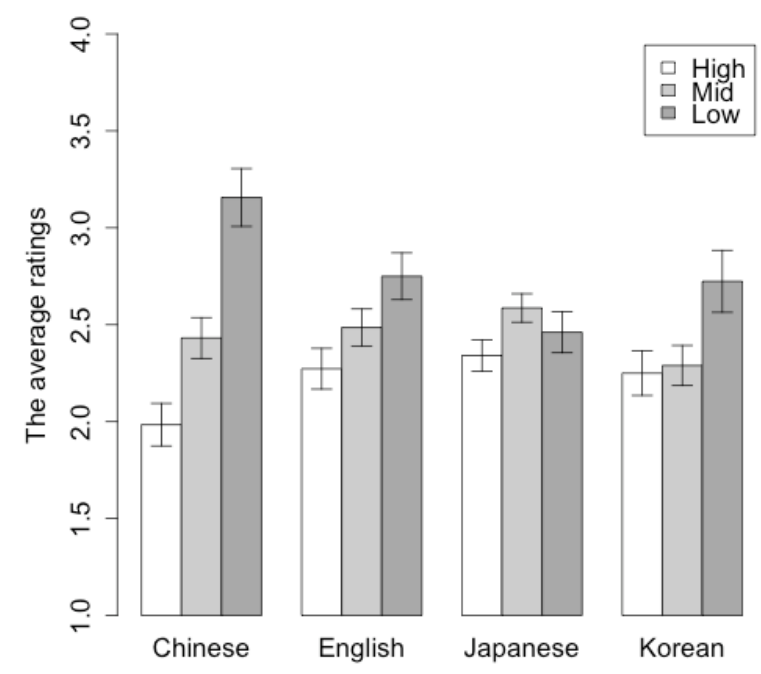

Figure 3 shows the effects of backness on the image of size. In all four languages, back vowels evoked significantly larger images than front vowels (Chinese, $t(798)=9.05, p<.001$; English, $t(878)=13.37, p<.001$; Japanese, $t(1678)=7.89$, $p<.001$; Korean, $t(758)=8.56, p<.001)$.

(6) Figure 3. The effect of vowel backness on size ratings.

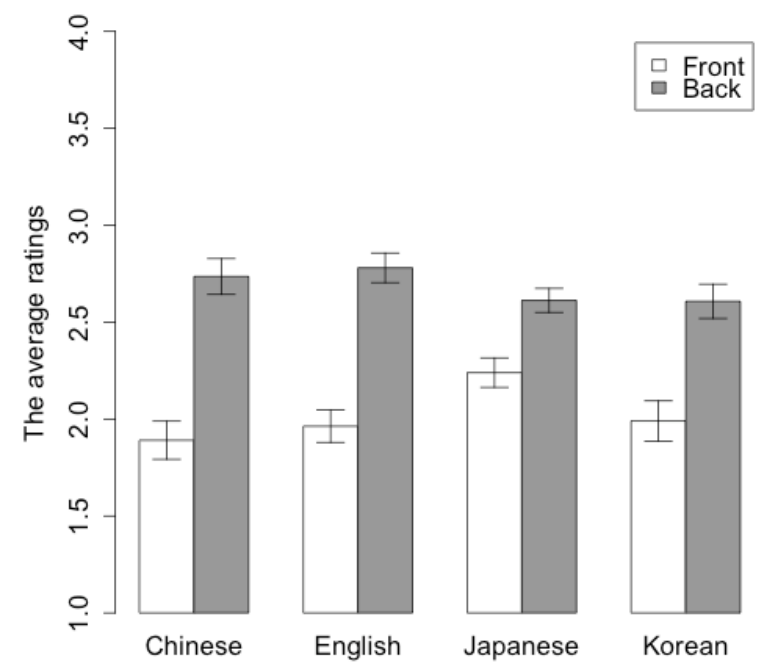

Figure 4 shows the effects of voicing of obstruents on the image of size. In Chinese, English, and Japanese, speakers rated voiced obstruents $[\mathrm{b}, \mathrm{d}, \mathrm{g}, \mathrm{z}]$ to be larger than voiceless obstruents [p, t, k, s] (Chinese, $t(798)=3.57, p<.001$; English, 
$t(878)=6.57, \quad p<.001 ;$ Japanese, $t(1678)=19.97, p<.001)$. Korean showed a non-significant reversal $(t(758)=-1.61$, n.s. $) .^{3}$

(7) Figure 4. The effect of obstruent voicing on size ratings.

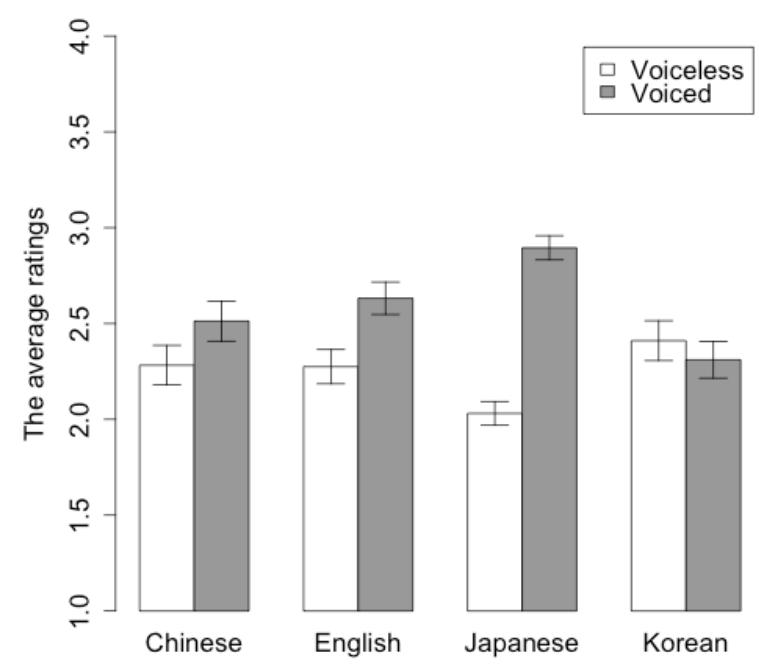

In summary, we find the following three general patterns: (i) low $>$ mid $>$ high, (ii) back $>$ front, and (iii) voiced $>$ voiceless.

\section{$5 \quad$ Discussion: Phonetic Grounding}

All three of the factors we have examined-vowel height, vowel backness, and voicing in obstruents - affect the image of size in all the four languages (with some exceptions). The next question is why these patterns hold cross-linguistically. We now discuss phonetic bases for these factors affecting the image of size by offering articulatory and acoustic explanations for each of the three factors.

\subsection{Vowel Height: An Articulatory Explanation}

We start with vowel height. Figure 5 illustrates the articulation of vowels at three different heights, in which the lower the vowel, the wider the aperture. This

\footnotetext{
${ }^{3}$ Young Ah Do (p.c.) suggested the following explanation for this exceptional behavior. In Korean, voiceless obstruents are realized as voiced in intervocalic position, and therefore Korean speakers may have perceived the voiceless stimuli (e.g. itit, ipip) as containing medial voiced consonants. Relatedly, Seunghun Lee (p.c.) shared his intuition that Korean speakers may be sensitive to size differences due to other laryngeal contrasts in such a way that aspirated consonants are larger than tense consonants, which are in turn larger than plain consonants.
} 


\section{Kazuko Shinohara and Shigeto Kawahara}

correlation may lead to the sensation of larger images in lower vowels.

(8) Figure 5. Articulatory configurations of vowels differing in height. The

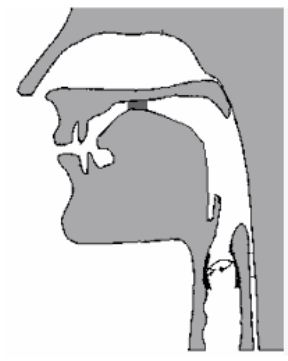

High vowel

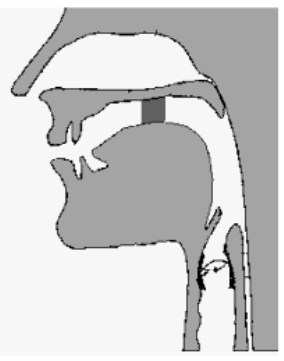

Mid vowel

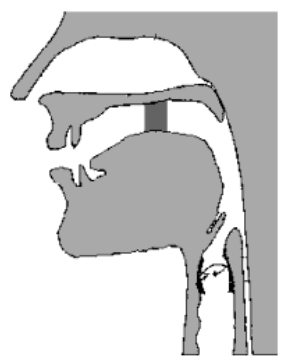

Low vowel thick vertical lines represent the degrees of aperture. $^{4}$

\subsection{Vowel Backness: An Articulatory Explanation}

Back vowels evoke larger images, presumably because they have a larger sub-oral cavity in front of the tongue. Figure 6 compares the oral cavities in front vowels and back vowels.

(9) Figure 6. Articulatory configuration of front and back vowels.

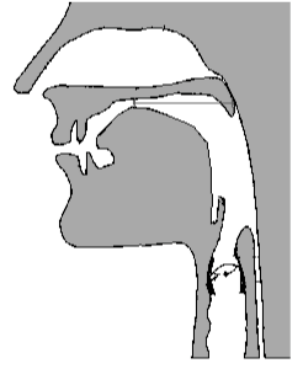

Front vowels

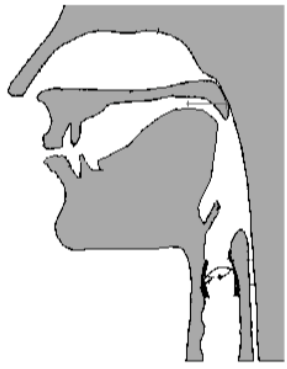

Back vowels

As illustrated in Figure 6, the sub-oral cavity in front of the tongue is larger in back vowels. Furthermore, non-low back vowels [u, o] are usually rounded, which enlarges the sub-oral cavity (Stevens et al. 1986). Speakers also lower their larynx in pronouncing back vowels to lengthen the entire oral cavity (Diehl and Kluender 1989: p.126 and references cited there). These articulatory gestures result in larger sub-oral cavities in front of the tongue, which in turn, may yield larger images.

\subsection{Vowel Features: An Acoustic Explanation}

The explanations so far are articulatory, but we can offer acoustic-based explana-

${ }^{4}$ Figures 5 and 6 are taken from http://www.ic.arizona.edu/ lsp/Phonetics/Vowels/. 


\section{A Cross-linguistic Study of Sound Symbolism}

tions based on the frequency code hypothesis (Ohala 1983b, 1994; see also Newman 1933; O'Boyle and Tarte 1980). This hypothesis builds on the correlation between the size of a resonator (or a resonating cavity) and its resulting frequency: lower frequencies imply large resonance cavities or resonators because the resonance frequency inversely correlates with its size.

We find that the second resonance frequency (F2) inversely correlates well with the judgments of size in our current experiment. Table 2 shows F2 values in Chinese (Howie 1976), English (Nishi et al. 2008), Japanese (Nishi et al. 2008), and Korean (Yang 1996). ${ }^{5}$ In Figure 1, the judged size of five vowels (roughly) follows the order of $[\mathrm{i}]<[\mathrm{e}]<[\mathrm{a}]<[\mathrm{u}]<[\mathrm{o}]$, and the $\mathrm{F} 2$ values (from high to low) follow the reserve order: $[\mathrm{i}]>[\mathrm{e}]>[\mathrm{a}]>[\mathrm{u}]>[\mathrm{o}]$ (with a reversal between the last two in Chinese).

(10) Table 2. F2 values of five vowels in four languages $(\mathrm{Hz})$.

\begin{tabular}{|l|r|r|r|r|r|}
\hline & \multicolumn{1}{|c|}{$\mathrm{i}$} & $\mathrm{e}$ & $\mathrm{a}$ & $\mathrm{u}$ & \multicolumn{1}{c|}{$\mathrm{o}$} \\
\hline Chinese & 2640 & 2200 & 1480 & 620 & 1080 \\
\hline English & 1805.5 & 1622.5 & 1210.5 & 1175.0 & 921.0 \\
\hline Japanese & 2076.5 & 1777.5 & 1158.0 & 1120.0 & 790.5 \\
\hline Korean & 2516.5 & 2172.5 & 1583.0 & 1001.0 & 987.0 \\
\hline
\end{tabular}

In general, then, F2 is a good predictor of the size of the images. One systematic exception is [a] in Chinese and Korean, which were judged to be the largest. It may be possible that [a] is considered to be large because [a] generally has a low F0 (Whalen and Levitt 1995) (for the discussion of F1, see subsection 5.6).

\subsection{Voicing in Consonants: An Articulatory Explanation}

We now turn to the effect of voiced obstruents, which are associated with large images. This association may have its roots in the articulation of voiced obstruents. Speakers expand their oral cavities when they pronounce voiced obstruents (Ohala 1983a), as illustrated in Figure 7. We can consider the entire oral cavity as consisting of two smaller spaces separated by the glottis: the oral cavity and the sub-glottal cavity. We call the air pressure in the oral cavity Po (for "intraoral air pressure") and the air pressure in the sub-glottal cavity Ps (for "subglottal air pressure") (Figure 7).

\footnotetext{
${ }^{5}$ The values for English, Japanese, and Korean are averaged over male and female speakers, while the Chinese data is based on one male speaker. To obtain data from comparable phonetic contexts, the values in Chinese are taken from those in the following context: in the $4^{\text {th }}$ tone (high-falling) syllable near the midpoint of the vowel; non-low back vowels had [w]-onset, front vowels had [y]-onset, and [a] had no onsets.
} 
(11) Figure 7. The oral cavity consisting of two sub-cavities separated by the glottis. 6

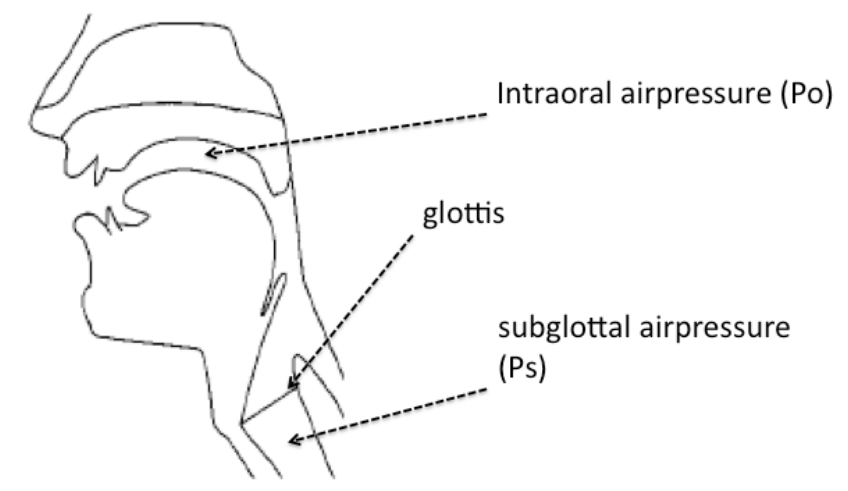

Po must be lower than Ps in order for the air to flow across the glottis. However, Po rises when the airway is significantly obstructed, which makes the condition Po $<$ Ps difficult to meet. In order to keep Po sufficiently low, speakers execute several articulatory maneuvers to expand their oral cavity, such as larynx lowering, velum raising, and cheek expansion (Ohala 1983a). This articulatory expansion of the oral cavity may lead to the sensation of large images.

\subsection{Voicing in Consonants: An Acoustic Explanation}

An acoustic explanation is also possible for the association between voiced obstruents and large images. Recall that a lower frequency implies larger objects (Ohala 1983b, 1994). Cross-linguistically, vowels have lower F0 next to voiced obstruents than next to voiceless obstruents (see Kingston and Diehl 1994 among others). Due to the lowering of F0, voiced obstruents may evoke larger images, because low F0 implies larger resonators.

\subsection{Summary: Articulatory or Acoustic?}

In summary, then, our results make either articulatory or acoustic sense. A question naturally arises at this point which type of explanation-articulatory or acoustic - better explains the sound symbolic patterns in natural languages. One challenge to the acoustic view is that we would have to postulate that speakers ignore F1, because low vowels have higher F1, yet evoke larger images. Another challenge may come from Eberhardt's (1940) finding that deaf children are sensitive to symbolic relations, although they showed behaviors slightly different from normal hearing children. She concludes that "while characteristic vowel

${ }^{6}$ The picture is taken from http://www.chass.utoronto.ca/ danhall/phonetics/. The illustration is ours. 


\section{A Cross-linguistic Study of Sound Symbolism}

frequency seemed to be the most important general factor involved in the choices of both deaf and normal subjects kinaesthetic data apparently played a relatively greater part in some cases with the deaf' (p. 36). At least deaf children seem to be sensitive to their articulatory gestures in the context of sound symbolism.

John Ohala (p.c.) pointed out however that the articulatory view alone cannot explain why high tones are sometimes associated with small images (e.g. in African languages: Ohala 1983a). He also points out that the articulatory view predicts that nasals may be associated with larger images because nasals include the nasal cavity in addition to the oral cavity. With these issues in mind, we would like to leave this question open at this point, and wait for future experimentation.

One line of research that could bear on this issue would consist of experiments using non-speech stimuli, which has been used to bear on the articulation/acoustic debate in other domains of phonetic theories - especially on the debate about the objects of speech perception (see Diehl et al. 2004 for a review). One such non-speech experiment on sound symbolism was conducted by O'Boyle and Tarte (1980), who did not find a significant correlation between frequencies of pure tones and the figures that they represent. They moreover cite (apparently unpublished) results (Tarte 1976) which found that speakers associated low tones with small figures significantly more often than with large objects-a correlation opposite of the one that the frequency code hypothesis predicts. More work using non-speech stimuli thus seems necessary.

Another line of approach may be to present to listeners non-native sounds, whose articulations cannot be guessed by the listeners. ${ }^{7}$ If the sound symbolic patterns have articulatory bases, then listeners would not be able to associate the non-native sounds with particular images. If sound symbolism patterns have psychoacoustic bases, then the listeners should have no trouble associating them to an image.

\subsection{A Final Issue: Inferences from the Lexicon?}

One final issue that we would like to raise here-without offering a definite answer - is whether our results can be explained based on inferences from existing lexical items. Stochastic patterns in the lexicon are known to affect our linguistic judgments (e.g. Hay et al. 2004). Appling this explanation to sound symbolism, for example, in English the word large has a low vowel; English also has a diminutive suffix $-y$ to represent small objects. From these lexical items, English speakers could have associated large images with low vowels and small images with a high front vowel $[i]^{8}$ (see also Ultan 1978 for an extensive cross-linguistic lexical study). However, such analogical lexicon-based explana-

\footnotetext{
${ }^{7}$ We would need to use those sounds that do not perceptually assimilate to native sounds.

${ }^{8}$ Alternatively, sound symbolism may diachronically affect the coinage of lexical words, skewing the lexicon in the direction that conforms to sound-symbolic patterns.
} 
tions face a non-negligible number of exceptions; e.g., small has a low vowel, big has a high front vowel, and huge has a high vowel.

Another systematic argument against this lexicon-based explanation comes from Korean. Korean has two sets of sound symbolic vowel categories, the bright category (e.g. [a, o]) and dark category (e.g. [u, i]) (Garrigues 1995: 367-371). Among other sound symbolic meanings, the bright category can denote "lightness, smallness and quickness" (Garrigues 1995: 368, citing Kim 1977: 69). Here non-high, back vowels correspond to the small images. It is unlikely therefore that the Korean participants in our experiment produced the results above based on inferences from existing items in their lexicon.

Although this Korean example is telling, it is beyond the scope of this short paper to make a definitive conclusion about a systematic lexicon-based explanation of sound symbolism. The question would ultimately boil down to: are the lexicons of the four languages we studied (and beyond) stochastically skewed enough to explain the sound symbolic patterns we observe? We would thus like to leave the testing of this question for future research.

\section{Summary and Conclusion}

Cross-linguistically, vowel height, backness, and voicing of obstruents all affect the perception of size. All of these patterns make phonetic sense (articulatory and/or acoustic). Speakers can project their articulatory gestures (or their acoustic consequences) to the sensation of image.

Patterns of sound symbolism can be a counterargument against the thesis of arbitrariness (Saussure 1916). Speakers have some non-arbitrary intuition about connections between sounds and meanings/images. They may have embodied motivations and may instantiate iconicity (e.g. Haiman 1983, 1985a, b) between sound and meaning. The study of sound symbolism thus can be an interesting topic in cognitive linguistics.

\section{Acknowledgments}

This research is partially supported by a Research Council Grant from Rutgers University to the second author. We thank Ayanna Beatie, Sophia Kao, and Allan Schwade for their help with data collection, and audiences at BLS 36, especially John Ohala, for their comments. Thanks are also due to Young Ah Do, Kelly Garvey, Shinsuke Oshida, Jeremy Perkins, Shanna Lichtman, Kyoko Yamaguchi, and especially Kimi Akita for their comments on earlier drafts of this paper.

\section{References}

Akita, Kimi. 2009. A Grammar of Sound-Symbolic Words in Japanese: Theoreti- 


\section{A Cross-linguistic Study of Sound Symbolism}

cal Approaches to Iconic and Lexical Properties of Mimetics. Ph.D. thesis, Kobe University, Kobe.

Baayen, Harald. 2008. Analyzing Linguistic Data: A Practical Introduction to Statistics Using R. Cambridge: Cambridge University Press.

Berlin, Brent. 2006. The First Congress of Ethnozoological Nomenclature. Journal of the Royal Anthropological Institute 12(1):23-44.

Diehl, Randay and K. R. Kluender. 1989. On the Objects of Speech Perception. Ecological Psychology 1(2):121-144.

Diehl, Randy L., A. J. Lotto, and L. L. Holt. 2004. Speech Perception. Annual Review of Psychology 55:149-179.

Eberhardt, Margarette. 1940. A Study of Phonetic Symbolism of Deaf Children. Psychological Monograph 52: 23-42.

Garrigues, Stephen L. 1995. Mimetic Parallels in Korean and Japanese. Studies in Language 19: 359-398.

Haiman, John. 1983. Iconic and Economic Motivation. Language 59: 781-819.

Haiman, John. 1985a. Natural Syntax. Cambridge: Cambridge University Press.

Haiman, John. ed. 1985b. Iconicity in Syntax. Amsterdam: John Benjamins.

Hay, Jennifer, P. Janet, and M. Beckman. 2004. Speech Perception, Well-formedness, and the Statistics of the Lexicon. Papers in Laboratory Phonology VI, 58-74. Cambridge University Press, Cambridge.

Howie, John Marshall (1976) Acoustical Studies of Mandarin Vowels and Tones. Cambridge: Cambridge University Press.

Johnson, Mark. 1987. The Body in the Mind: The Bodily Basis of Meaning, Imagination, and Reason. Chicago: University of Chicago Press.

Kawahara, Shigeto, Y. Matsunaka, A. Nakayama, and K. Shinohara. 2005. An Experimental Case Study of Sound Symbolism in Japanese. A paper presented at New Directions in Cognitive Linguistics Conference. University of Sussex, UK. 
Kazuko Shinohara and Shigeto Kawahara

Kim, Kong-On. 1977. Sound Symbolism in Korean. Journal of Linguistics 13: 67-75.

Kingston, John, and R. Diehl. 1994. Phonetic Knowledge. Language 70:419-454.

Kunihira, Shirou. 1971. Effects of the Expressive Force on Phonetic Symbolism. Journal of Verbal Learning and Verbal Behavior 10:427-429.

Lakoff, George, and M. Johnson. 1980. Metaphors We Live By. Chicago: Chicago University Press.

Lakoff, George, and M. Johnson. 1999. Philosophy in the Flesh. New York: Basic Books.

MacNeilage, Peter, and B. L. Davis. 2001. Motor Mechanisms in Speech Ontogeny: Phylogenetic, Neurobiological and Linguistic Implications. Current Biology 11: 696-700.

Newman, S. 1933. Further Experiments in Phonetic Symbolism. American Journal of Psychology 45: 53-75.

Nishi, Kanae, W. Strange, R. Akahane-Yamada, R. Kubo, and S. A. Trent-Brown. 2008. Acoustic and Perceptual Similarity of Japanese and American English Vowels. Journal of Acoustical Society of America 124:576-588.

O'Boyle, Michael, J. and R. D. Tarte. 1980. Implications for Phonetic Sound Symbolism: The Relationship between Pure Tones and Geometric Features. Journal of Psycholinguistic Research 9(6): 535-544.

Ohala, John, J. 1983a. The Origin of Sound Patterns in Vocal Tract Constraints. In P. MacNeilage ed., The Production of Speech, 189-216. Berlin: Springer-Verlag.

Ohala, John, J. 1983b. The Phonological Ends Justify Any Means. In S. Hattori and K. Inoue, eds., Proceedings of the XIIIth International Congress of Linguists 1982, 232-243. Tokyo: Sanseido Shoten.

Ohala, John, J. 1994. The Frequency Codes Underlies the Sound Symbolic Use of Voice Pitch. In L. Hinton, J. Nichols, and J. J. Ohala, eds., Sound Symbolism, 325-347. Cambridge: Cambridge University Press.

Paget, Richard 1930. Human Speech: Some Observations, Experiments, and Conclusions as to the Nature, Origin, Purpose, and Possible Improvement of 


\section{A Cross-linguistic Study of Sound Symbolism}

Human Speech. London: Routledge.

Sapir, Edward. 1929. A Study in Phonetic Symbolism. Journal of Experimental Psychology 12:225-239.

Saussure, Ferdinand. de. 1916. Cours de Linguistique Générale. C. Bally, A. Sechehaye and A. Riedlinger, eds. Paris: Payot.

Stevens, Kenneth, S. J. Keyser, and H. Kawasaki. 1986. Toward a Phonetic and Phonological Theory of Redundant Features. In J. S. Perkell and D. H. Klatt eds., Invariance and Variability in Speech Processes, 426-449. Hillsdale, New Jersey: Lawrence Erlbaum.

Tarte, D. Robert. 1976. Phonetic Sound Symbolism for Pure Tones. A paper presented at the 17th Meeting of Psychonomic Society, St. Louis, Missouri.

Ultan, Russel. 1978. Size-Sound Symbolism. In J. Greenberg ed., Universals of Human Language II: Phonology, 525-568. Stanford: Stanford Univ. Press.

Whalen, Douglas, and A. G. Levitt. 1995. The Universality of Intrinsic F0 of Vowels. Journal of Phonetics 23, 349-366.

Yang, Byunggon. 1996. A Comparative Study of American English and Korean Vowels Produced by Male and Female Speakers. Journal of Phonetics 24: 245-261.

Kazuko Shinohara

Tokyo University of Agriculture and Technology

2-24-16 Nakacho, Koganei

Tokyo 184-8588, JAPAN

k-shino@cc.tuat.ac.jp

Shigeto Kawahara

Keio University

2-15-45

Mita, Minato-ku

Tokyo, JAPAN

kawahara@icl.keio.ac.jp 